Series A

\author{
I. MATHEMATICA
}

340

\title{
ON HOMOLOGY THEORIES IN LOCALLY CONNECTED SPACES
}

\author{
BY \\ OLLI JUSSILA
}


Communicated 9 April 1963 by P. J. Myrberg and Olli Lehto 


\section{Introduction}

In this paper we use the method of normal refinements to derive the results of Mardešic [6] simultaneously for many homology theories. The notations and results of $\S 2$ in [3] are assumed.

Section 1 introduces the homology theories used here and the second section presents a uniqueness theorem for them. In the third section similar results are outlined for relative homology. The last section is devoted to some applications to paracompact spaces. The appendix contains some corrections to my previous paper [3].

\section{§1. Fine Complexes}

1. Let $Z$ denote the ring of integers. $R$ shall be a fixed ring, $M_{R}$ the category of $R$-modules, and $C_{R}$ the category of augmented chain complexes in $M_{R}$.

Let $X$ be a set. The set $P(X)$ of subsets of $X$ together with the inclusions $i^{P, Q}: P \rightarrow Q(P \subset Q \subset X)$ is a category (cf. [2], p. 16). If $X^{\prime} \subset X$, then all functors of $P(X)$ are identified on $P\left(X^{\prime}\right)$ with their restrictions to $P\left(X^{\prime}\right)$.

Definition 1. A complex $C$ on $X$ is a covariant functor $P(X) \rightarrow C_{R}$, i.e. the value of $C$ on $P \subset X$ is an augmented chain complex $C(P)=$ $\left(C_{n}(P)\right)_{n \in Z}$. The $n$-dimensional homologyfunctor $H_{n}$ of $C$ is the covariant functor $P(X) \rightarrow M_{R}$, whose value $H_{n}(P)$ on $P \subset X$ is the module $H_{n}(C(P))$, the functorial homomorphisms

$$
H_{n}\left(i^{P, Q}\right): H_{n}(P) \rightarrow H_{n}(Q) \quad(P \subset Q \subset X)
$$

being induced by the functorial homomorphisms

$$
C\left(i^{P, Q}\right): C(P) \rightarrow C(Q) \text {. }
$$

2. A covering $\alpha=\left(A_{i}\right)_{i \in I}$ of $X$ is a family of subsets of $X$, whose union is $X$. The covering $\alpha$ is said to be proper, if $A_{\boldsymbol{i}}=A_{\boldsymbol{j}}$ implies $i=j(i, j \in I)$. If $s \subset \alpha, s \neq \varnothing$, we shall denote by $\hat{s}$ the intersection of the members of $s$. If $A \subset X$ then $\alpha \cap A$ is the 
covering $\left(A_{i} \cap A\right)_{i \in I}$ of $A$. If $\varepsilon=\left(E_{j}\right)_{j \in J}$ is another covering of $X$, then $\alpha \cap \varepsilon$ stands for the covering $\left(A_{i} \cap E_{j}\right)_{i \in I, j \in J}$ of $X$.

If $\alpha$ is a covering of $X$ and $C$ a complex on $X$, we shall denote by $\stackrel{\alpha}{C}_{p, q}(p, q \in Z)$ (i) the zero-module, if $p<-1$, (ii) $C_{q}(X)$, if $p=-1$ and (iii) the direct sum $\sum_{i_{0}, \ldots i_{p} \in I} C_{q}\left(A_{i_{0}} \cap \ldots \cap A_{i_{p}}\right)$, if $p>-1$. Let $\varphi_{k}(k=0, \ldots, p)$ denote the functorial homomorphism

$$
C_{q}\left(A_{i_{0}} \cap \ldots \cap A_{i_{p}}\right) \rightarrow C_{q}\left(A_{i_{0}} \cap \ldots \cap \hat{A}_{i_{k}} \cap \ldots \cap A_{i_{p}}\right)
$$

for $p>0$, and

$$
C_{q}\left(A_{i_{0}}\right) \rightarrow C_{q}(X)
$$

for $p=0$. If $p \geq 0$, we define a homomorphism

$$
\partial^{\prime}: \stackrel{\alpha}{C_{p, q}} \rightarrow \stackrel{\alpha}{C}_{p-1, q} \quad(q \in Z)
$$

by

$$
\partial^{\prime}(c)=\sum_{k=0}^{p}(-1)^{k} \varphi_{k}(c)
$$

for $c \in C_{q}\left(A_{i_{0}} \cap \ldots \cap A_{i_{p}}\right)$. It is easy to see that $\partial^{\prime} \partial^{\prime}=0$. The' family $\stackrel{\alpha}{C}_{* q}=\left(\stackrel{\alpha}{C_{p}, q}\right)_{p \in Z}$ becomes thus an augmented chain complex. We shall denote by $H_{p}(\alpha)$ the module $H_{p}\left({ }^{\prime} C_{*-1}\right)$ for each $p \in Z$.

Let $\beta$ be another covering of $X$. The covering $\alpha$ is said to be a refinement of $\beta$, if there exists a mapping $\pi^{\alpha \beta}: \alpha \rightarrow \beta$, called projection, such that $A_{i} \subset \pi^{\alpha \beta}\left(A_{i}\right)$ for each $A_{i} \in \alpha$. We shall then write $\alpha>\beta$. The projection $\pi^{\alpha \beta}$ induces for each $q \in Z$ a homomorphism

$$
\pi_{* q}^{\alpha \beta}: \stackrel{\alpha}{C}_{* q} \rightarrow{ }^{\prime} \stackrel{\beta}{C}_{* q}
$$

with components

$$
\pi_{p, q}^{\alpha \beta}: \stackrel{\alpha}{C_{p, q}} \rightarrow \stackrel{\beta}{C}_{p, q} \quad(p \in Z)
$$

defined by

$$
\pi_{p, q}^{\alpha \beta}(c)=C_{q}\left(\hat{i s}, \widehat{\pi^{\alpha \beta}(s)}\right)(c)
$$

and linearity $\left(c \in C_{q}\left(A_{i_{0}} \cap \ldots \cap A_{i_{p}}\right), s=\left(A_{i_{0}}, \ldots, A_{i_{p}}\right)\right)$. We shall denote by $\pi_{(p) q}^{\alpha \beta}$ the functorial homomorphism

$$
H_{p}\left(\pi_{* q}^{\alpha \beta}\right): H_{p}\left({ }^{\prime} \stackrel{\alpha}{C}_{* q}\right) \rightarrow H_{p}\left({ }^{\prime} \stackrel{\beta}{C}_{* q}\right)
$$

and by $\pi_{(p)}^{\alpha \beta}$ the homomorphism $\pi_{(p)-1}^{\alpha \beta}: H_{p}(\alpha) \rightarrow H_{p}(\beta) \quad(p, q \in Z)$. 
3. The differentials

$$
\partial: C_{q}\left(A_{i_{0}} \cap \ldots \cap A_{i_{p}}\right) \rightarrow C_{q-1}\left(A_{i_{0}} \cap \ldots \cap A_{i_{p}}\right)
$$

of $C\left(A_{i_{0}} \cap \ldots \cap A_{i_{p}}\right)$ commute with the differentials (1). If we denote by $(-1)^{p+1} \partial^{\prime \prime}$ the homomorphism of $\stackrel{\alpha}{C}_{p, q}$ into $\stackrel{\alpha}{C_{p, q-1}}$ induced by the homomorphisms (3), it follows that the homomorphisms $\partial^{\prime}$ and $\partial^{\prime \prime}$ make the family $\stackrel{\alpha}{C_{*}}=\left(\stackrel{\alpha}{C_{p}, q}\right)_{p, q \in Z}$ an augmented double chain complex (see [3], p. 21). It follows immediately from the definitions (1), (2) and (3), that the homomorphisms $\pi_{p, q}^{\alpha \beta}, \partial^{\prime}$ and $(-1)^{p+1} \partial^{\prime \prime}$ commute with each other. Hence they induce functorially a homomorphism $\pi^{\alpha \beta}: \stackrel{\alpha}{C_{*}} \rightarrow \stackrel{\beta}{C_{*}}$ of augmented double chain complexes, which coincides with $\pi_{*-1}^{\alpha \beta}$ on ${ }^{\prime} \stackrel{\alpha}{*-1}_{\text {and }}$ an the identity map on $\stackrel{\alpha}{C_{-1 *}}={ }^{\prime \prime} C_{-1 *}$.

Definition 2. The complex $C$ is said to be fine with respect to $\alpha$, if $\stackrel{\alpha}{C}_{* q}$ is acyclic for $q>-1$; i.e., if $H_{p}\left({ }^{\prime} C_{* q}\right)=0$ for each $p \in Z$ and $q<-1$.

Henceforth we consider only those coverings with respect to which $C$ is fine; this is equivalent to condition (2.2) of [3] on page 22. We obtain thus the canonical homomorphisms

$$
h_{n}^{\alpha}: H_{n}(X)=H_{n}\left({ }^{\prime \prime} \stackrel{\alpha}{Z}_{-1 *}\right) \stackrel{D_{n}}{\longrightarrow} H_{-1}\left({ }^{\prime \prime} \stackrel{\alpha}{Z}_{n *}\right) \stackrel{j}{\longrightarrow} H_{n}\left({ }^{\prime} \stackrel{\alpha}{C}_{*-1}\right)=H_{n}(\alpha),
$$

where $j$ is a canonical epimorphism (see [3], pp. 22, 29). The equation

$$
h_{n}^{\beta}=\pi_{(n)}^{\alpha \beta} h_{n}^{\alpha}
$$

follows immediately from the definitions.

Definition 3. The coverings $\alpha$ and $\beta$ are said to determine $H_{n}(X)$, if $h_{n}^{\beta}$ is an isomorphism onto $\pi_{(n)}^{\alpha \beta}\left(H_{n}(\alpha)\right)$ (see [1], p. 320).

Definition 4. (cf. [5], p. 214) A net of $X$ is a set $N$ of coverings of $X$, directed by the relation $<$. For each $n \in Z$ we shall denote by $H_{n}(N)$ the inverse limit of the modules $H_{n}(\alpha) \quad(\alpha \in N)$ under the homomorphisms $\pi_{(n)}^{\alpha \beta}$ and by $\pi_{(n)}^{\beta}: H_{n}(N) \rightarrow H_{n}(\beta)$ the inverse limit of the homomorphisms $\pi_{(n)}^{\alpha \beta}(\alpha \in N)$. The equations (5) give rise to limit homomorphisms

$$
t_{n}: H_{n}(X) \rightarrow H_{n}(N) \quad(n \in Z)
$$

of the homomorphisms $h_{n}^{\alpha}(\alpha \in N)$ satisfying the equations

$$
h_{n}^{\alpha}=\pi_{(n)}^{\alpha} t_{n} \quad(\alpha \in N, n \in Z) .
$$


Examples: $1^{\circ}$ Let $X$ be a topological space, $A$ a subspace of $X$, $M$ a module and $\alpha$ an open covering of $X$. If $P \subset X$, let $C^{A}(P) \quad$ be the augmented complex $S(P, P \cap A) \otimes M$ of singular chains of $(P, P \cap A)$ with coefficients in $M$ and $C^{\alpha A}(P)$ the subcomplex $S_{\alpha}(P, P \cap A) \otimes M$ of $C^{A}(P)$ generated by singular simplices $s$, for which one can find an element of $\alpha$ which contains the carrier $\|s\|$ of $s \quad$ (see [6], p. 152). Let $T_{n}^{P}$ be the set of those generators of $C_{n}^{\alpha A}(P)$ whose carriers are not in $A$. Then $C_{n}^{\alpha A}(P)$ is canonically isomorphic with a direct sum $\sum_{s \in T_{n}^{P}} M_{s}$ of modules $M_{s}$ isomorphic with $M$. If $P \subset Q \subset X$, then the functorial homomorphism $C_{n}^{\alpha A}(P) \rightarrow C_{n}^{\alpha A}(Q)$ is induced by the inclusion $T_{n}^{P} \subset T_{n}^{Q}$. Thus ${ }^{\prime}{\stackrel{\alpha}{C_{n}} \alpha A}_{(n>-1)}$ is canonically isomorphic with a direct sum $\sum_{s \in T} \underset{n}{X}\left(\alpha^{s}, M_{s}\right)$, where $\alpha^{s}$ is the closed subcomplex of the nerve of $\alpha$ composed of those members of $\alpha$ which contain $\|s\|$. Because the complexes $\alpha^{s}$ are simplices, it follows that $C^{\alpha \mathrm{A}}$ is fine with respect to $\alpha$ (cf. [4], p. 706).

We have by definition $C_{-1}^{\alpha \mathrm{A}}(P)=M$ for $P \neq \varnothing$ and $P \cap A=\varnothing$ and $C_{-1}^{\alpha \mathrm{A}}(P)=0 \quad$ otherwise. Thus ${ }^{\prime} C_{-1}^{\alpha \mathrm{A}}$ is isomorphic with the augmented complex $C\left(X_{\alpha}, A_{\alpha}, M\right)$ of the $M$-valued chains of the nerve of $\alpha$ modulo $A$. On the other hand it is well known that the inclusion $C^{\alpha \mathrm{A}}(P) \subset C^{\mathrm{A}}(P)$ induces a homotopy equivalence of complexes for each $P \subset X$ and for each open covering $\alpha$ of $X$. These facts will be used in the last paragraph.

$2^{\circ}$ As in [3], pp. 10, 14, let $M$ be a covariant functor $P(X) \rightarrow M_{R}$ such that $M(\emptyset)=0$; let $S$ be a spectrum of $X$ and $\alpha$ a finite covering of $X$ belonging to $S$. Then $S$ and $M$ define a complex $C^{S}$ on $X$, whose components $C_{n}^{S}(n \in Z)$ are the functors $C_{n}(S, \stackrel{\circ}{M})$ (see [3], p. 17). We have shown in [3], pp. 28-29, that $C^{S}$ is fine with respect to $\alpha$ (on page $29, \phi$ should be added in front of $c_{m}^{\beta},-c_{m}^{\beta}$ and $-c_{m k}^{\beta}$ in the equations (3.4) and (3.5)).

If $\beta$ is a finite covering of $X$, we may use $S$ to construct a new spectrum which contains $\beta$, as follows: Let $S \cap \beta$ be the set $\{\alpha \cap \beta ; \alpha \in S\}$. If $\alpha>\alpha^{\prime}\left(\alpha, \alpha^{\prime} \in S\right)$ and $\pi^{\alpha \alpha^{\prime}}$ is the corresponding projection belonging to $S$, we define a projection $\pi$ of $\alpha \cap \beta$ in $\alpha^{\prime} \cap \beta$ by $\pi\left(A_{i} \cap B_{j}\right)=\pi^{\alpha \alpha^{\prime}}\left(A_{i}\right) \cap B_{j} \quad\left(A_{i} \in \alpha, B_{j} \in \beta\right)$. Then $S \cap \beta$ with these projections is a spectrum which contains $\beta$.

If $\beta$ has a refinement $\gamma$ in $S$, then $C^{S}$ is fine with respect to $\beta$. In fact, without changing the complex $C^{S}$ on $X$, we may substitute for $S$ the spectrum $S(\beta)=\{\alpha>\gamma ; \alpha \in S\} \cup \beta$, where $\pi^{\gamma \beta}: \gamma \rightarrow \beta$ is fixed arbitrarily and $\pi^{\alpha \beta}: \alpha \rightarrow \beta$ is the composed projection $\pi^{\gamma \beta} \pi^{\alpha \gamma}, \pi^{\alpha \gamma}$ belonging to $S$. 
Remark: If $X$ is a topological space, then the spectra in the examples $A-D$ of [3], p. 15, are rather uninteresting from the homological viewpoint, because $C_{n}(U(X), M \mid P)=C_{n}(F(X), M \mid P)=0$ for $n>0$ and for each $P \subset X$. Instead the grating spectrum $\Sigma$ of $X$ yields non-trivial homology theories (cf. [5], p. 279).

We take this opportunity to note that remark $2^{\circ}$ in $[3]$, p. 32 , can be corrected by transferring it into cohomological form. The theorem of Floyd then follows by Pontryagin duality.

\section{§2. n-refinements}

4. Let $\alpha$ and $\beta$ be two coverings of $X$, and $N$ a net of $X$.

Definition 5. (cf. [1], p. 320) The covering $\alpha$ is said to be a (strong) $n$-refinement of $\beta$ if there exists a projection $\pi^{\alpha \beta}: \alpha \rightarrow \beta$ such that

$$
\begin{aligned}
& \operatorname{Im}\left(H_{n}\left(i^{A_{i}, \pi^{\alpha \beta}\left(A_{i}\right)}\right): H_{n}\left(A_{i}\right) \rightarrow H_{n}\left(\pi^{\alpha \beta}\left(A_{i}\right)\right)\right)=0 \\
& \left(\operatorname{Im}\left(H_{k}\left(\widehat{i^{s}, \widehat{\pi^{\alpha \beta}(s)}}\right): H_{k}(\widehat{s}) \rightarrow H_{k}\left(\widehat{\pi^{\alpha \beta}(s)}\right)\right)=0\right)
\end{aligned}
$$

for each $A_{i} \in \alpha \quad$ (for each $s \subset \alpha$ and $k<n+1$ ). We shall then write $\alpha \mid n>\beta \quad(\alpha \mid n \gg \beta)$.

Definition 6. The net $N$ is said to be semi-lc if there exists an element of $N$ which has an $n$-refinement in $N ; N$ is said to be $l c_{n}$ if every element of $N$ has a strong $n$-refinement in $N$.

Lemma 1. If ${ }^{n}|n-2 \gg \ldots| n-2 \gg{ }^{0} \mid n-1>{ }^{-1} \quad{ }^{i}(\alpha \in N ; i=$ $-1,0, \ldots, n),{ }_{i}{ }_{i} h_{i-1}^{n}$ is injective and $\operatorname{Im}\left(h_{n}^{\alpha}\right)=\operatorname{Im}\left(\pi_{(n)}^{\alpha, \alpha}\right)$.

Proof. Let ${ }^{i}: \alpha \rightarrow \alpha(i=0, \ldots, n)$ be the projections defining the relations $\mid n-2 \gg$ and $|n-1\rangle$ above and $\pi$ the composite projection $\pi{ }_{0} 1 \ldots \pi$. For each $k \in Z$ we have the commutative diagram

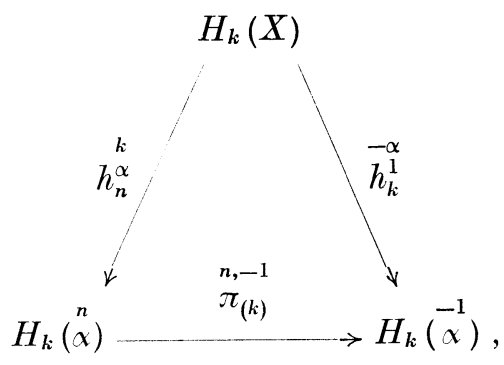


which is identical with the commutative diagram

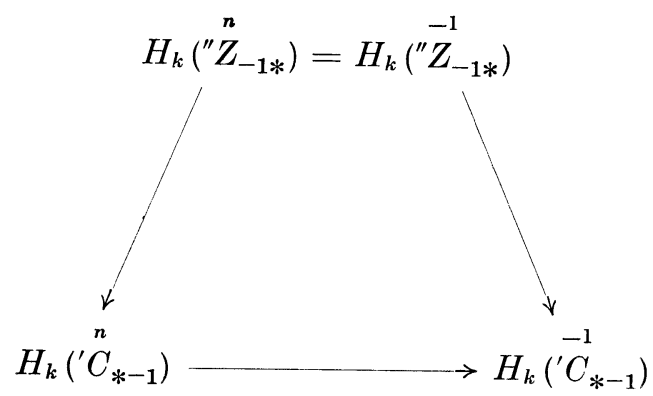

of the corresponding double complexes $\stackrel{i}{C_{*}}=\stackrel{\alpha^{i}}{C_{*}}(-1 \leq i \leq n)$. In virtue of the assumption the image of the canonical homomorphism

$$
H_{n-k-1}\left("{ }^{\prime \prime} C_{k *}\right) \rightarrow H_{n-k-1}\left({ }^{k-1} C_{k *}\right),
$$

induced by the homomorphism ${ }^{k} \pi_{*}$, is zero for each $k=0, \ldots, n$. Setting $k=n$ in (8) we see as in [3] p. 31, that

$$
\begin{aligned}
& \operatorname{Ker}\left(H_{-1}\left({ }^{\prime \prime} Z_{n-1 *}^{n}\right) \stackrel{j}{\longrightarrow} H_{n-1}\left({ }^{\prime} C_{*-1}\right)\right) \subset \\
& \operatorname{Ker}\left(H_{-1}\left({ }^{\prime \prime} Z_{n-1 *}^{n}\right) \longrightarrow H_{-1}\left({ }^{\prime \prime} Z_{n-1 *}\right)\right) .
\end{aligned}
$$

Theorem 1 and Corollary 1 of [3], pp. 25, 26, then imply the assertion.

Corollary 1. If $\alpha^{2 n+2}|n-1 \gg \ldots| n-1 \gg \alpha^{0} \mid n>\alpha^{-1}$, then $\alpha^{2 n+2}$ and $\alpha^{n+1}$ determine $H_{p}(X)$ for each $p<n+1$.

Theorem 1. If a net $N$ of $X$ is $l c_{n-1}$ and semi- $l c_{n}$, then the homomorphism $t_{p}: H_{p}(X) \rightarrow H_{p}(N)$ is bijective for each $p<n-1$.

Proof: In virtue of Corollary $1, h_{p}^{\alpha}$ is an isomorphism onto $\pi_{(p)}^{\alpha}\left(H_{p}(N)\right)$ for each sufficiently fine element $\alpha$ of $\Lambda$. If $\beta>\alpha$ $(\beta \in N)$, the relations (5) imply that

$$
\pi_{(p)}^{\beta \alpha}: \pi_{(p)}^{\beta}\left(H_{p}(N)\right) \rightarrow \pi_{(p)}^{\alpha}\left(H_{p}(N)\right)
$$

is bijective. Because $H_{p}(N)$ is the inverse limit of the modules $\pi_{(p)}^{\alpha}\left(H_{p}(N)\right)$ under the homomorphisms $\pi_{(p)}^{\beta \alpha}$, the theorem follows from the equations (7). 


\section{§3. Relative homology}

5. In this section we shall indicate briefly how the ideas above can be transferred to relative homology. We shall suppose that $C\left(i^{P, Q}\right): C(P) \rightarrow$ $C(Q)$ defines $C(P)$ as a subcomplex of $C(Q)$ for all $P \subset Q \subset X$. Let $A$ be a fixed subset of $X$. Setting $H_{n}(X, A)=H_{n}(C(X) / C(A))$ for each $n \in Z$, we obtain the exact homology sequence $S_{C}$ of $C$ :

$$
S_{C}: \ldots H_{n}(A) \rightarrow H_{n}(X) \rightarrow H_{n}(X, A) \rightarrow H_{n-1}(A) \rightarrow \ldots
$$

A covering $\alpha=\left(\alpha_{1}, \alpha_{2}\right)$ of $(X, A)$ is a covering $\alpha_{1}$ of $X$ and a subfamily $\alpha_{2}$ of $\alpha_{1}$, whose members cover $A$ (cf. [6], p. 151). If $\beta=\left(\beta_{1}, \beta_{2}\right)$ is another covering of $(X, A)$, then a projection $\pi^{\alpha \beta}$ : $\alpha \rightarrow \beta$ is a projection $\alpha_{1} \rightarrow \beta_{1}$ such that $\pi^{\alpha \beta}\left(\alpha_{2}\right) \subset \beta_{2}$. The covering $x$ is then said to be a refinement of $\beta$ (in symbols $\alpha>\beta$ ).

Henceforth we shall write ${\stackrel{\alpha}{C_{2}}}_{*}$ and $H_{n}\left(\alpha_{2}\right)$ for $C_{*}$ and $H_{n}\left(\alpha_{2} \cap A\right)$ respectively. Then $C_{\alpha_{1}}^{\alpha_{2}}$ may be identified with a subcomplex of $C_{*}$. Writing $H_{n}(\alpha)=H_{n}\left({ }^{\prime} C_{*-1} / C_{*-1}\right)$ for each $n \in Z$ we obtain the exact homology sequences $S_{\alpha}$ and $S_{\beta}$ below. The projection $\pi^{\alpha \beta}$ induces a homomorphism $\pi_{S}^{\alpha \beta}$ of $S_{\alpha}$ into $S_{\beta}$, which is represented by the commutative diagram

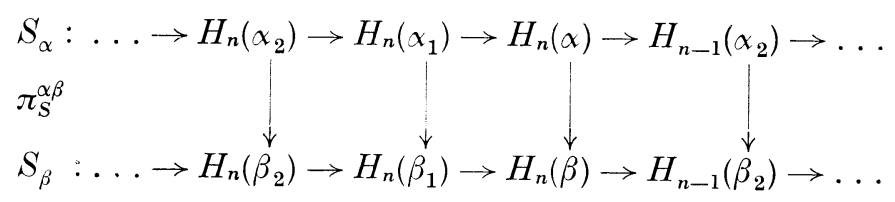

The complexes $\quad{ }^{\alpha_{1}} C_{* q}$ and ${ }^{\alpha_{2}} C_{* q}$ are by assumption acyclic for $q>-1$. Denoting by $\stackrel{\alpha}{C_{*}}$ the quotient complex ${\stackrel{\alpha}{C_{1}}}_{*}^{\alpha_{2}} C_{*}$, it follows that ${ }^{\prime} C_{* q}^{\alpha}={ }^{\prime} C_{* q}{ }^{\prime} C_{* q}$ is acyclic for $q>-1$. We obtain in this way the homomorphisms $h_{S}^{\alpha}: S_{C} \rightarrow S_{\alpha}$ such that

$$
h_{S}^{\beta}=\pi_{S}^{\alpha \beta} h_{S}^{\alpha} .
$$

A net of $(X, A)$ is a set $N$ of coverings of $(X, A)$ directed by the relation $<$. The coverings $\alpha_{1}$ and $\alpha_{2} \cap A(\alpha \in N)$ are the elements of the nets $N_{1}$ and $N_{2}$ of $X$ and $A$ respectively. Let $H_{n}(N)$ be the inverse limit of the modules $H_{n}(\alpha)(\alpha \in N)$ induced by the projections. In general the limit sequence

$$
S_{N}: \ldots \rightarrow H_{n}\left(N_{2}\right) \rightarrow H_{n}\left(N_{1}\right) \rightarrow H_{n}(N) \rightarrow H_{n-1}\left(N_{2}\right) \rightarrow \ldots
$$


of the sequences $S_{\alpha}(\alpha \in N)$ is not exact. If $\pi_{S}^{\beta}: S_{N} \rightarrow S_{\beta}$ is the limit homomorphism of the homomorphisms $\pi_{S}^{\alpha \beta}(\alpha \in N, \alpha>\beta)$, the equations (10) give rise the a homomorphism $t_{S}: S_{C} \rightarrow S_{N}$ such that

$$
h_{S}^{\alpha}=\pi_{S}^{\alpha} t_{S}
$$

for each $\alpha \in N$.

6. If $N_{1}$ and $N_{2}$ are $l c_{n-1}$ and semi- $l c_{n}$, there exists for each sufficiently fine $\delta \in N$ a sequence $\alpha>\beta>\gamma>\delta$ of elements of $N$, where $\alpha_{2} \cap A$ and $\beta_{2} \cap A, \beta_{2} \cap A$ and $\gamma_{2} \cap A$ as well as $\gamma_{2} \cap A$ and $\delta_{2} \cap A$ determine $H_{p}(A)$ for each $p<n+1$ and $\alpha_{1}$ and $\beta_{1}, \beta_{1}$ and $\gamma_{1}$ as well as $\gamma_{1}$ and $\delta_{1}$ determine $H_{p}(X)$ for each $p<n+1$. Consider the following part of the composite homomorphism $\pi_{S}^{\gamma \delta} \pi_{S}^{\beta \gamma} \pi_{S}^{\alpha \beta} h_{S}^{\alpha}$ :

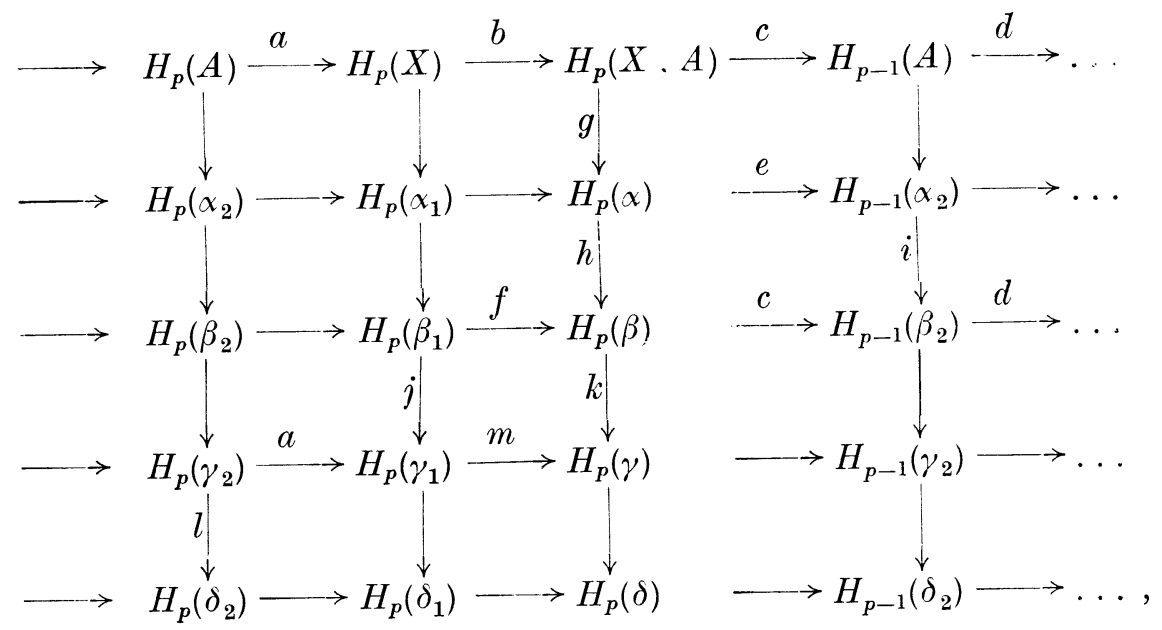

where $p<n+1$ and $H_{p}(A), H_{p}(X)$ and $H_{p-1}(A)$ are identified canonically with a common submodule of the modules of the corresponding vertical rows. The letters $a$ to $m$ are symbols of homomorphisms already defined above.

We show first, that $k h g$ is injective. If $x \in H_{p}(X, A)$ and $(k h g)(x)$ $=0$, it follows that $c(x)=0$. There exists a $y \in H_{p}(X)$ such that $b(y)=x$. Then $m(y)=0$. There exists a $z \in H_{p}\left(\gamma_{2}\right)$ such that $a(z)=y$. Then $l(z) \in H_{p}(A), \quad(a l)(z)=y$ and $(b a l)(z)=x=0$. Thus $H_{p}(X, A)$ may be identified with a common submodule of $H_{p}(\alpha), H_{p}(\beta)$ and $H_{p}(\gamma)$. We will show that $\pi_{(p)}^{\alpha \gamma}\left(H_{p}(\alpha)\right)=H_{p}(X, A)$. If $x \in H_{p}(\alpha)$, then $(i e)(x)$ $\in H_{p-1}(A)$ and $($ die $)(x)=0$. There exists a $y \in H_{p}(X, A)$ such that $c(y)=(i e)(x)$; i.e. $\quad c(h(x)-y)=0$. There exists a $z \in H_{p}\left(\beta_{1}\right)$ such that $f(z)=h(x)-y$. Then $j(z) \in H_{p}^{-}(X)$ and $(m j)(z)+y=(k h)(x)$ $\in H_{p}(X, A)$. 
We have thus shown that $\alpha$ and $\gamma$ determine $H_{p}(X, A)$ for each $p<n+1$. Then a similar proof as in Theorem 1 yields

Theorem 2. If $N_{1}$ and $N_{2}$ are $l c_{n-1}$ and semi-lc $c_{n}$, then the homomorphism

$$
t_{p}: H_{p}(X, A) \rightarrow H_{p}(N)
$$

of $t_{S}$ is bijective for each $p<n+1$.

\section{§4. Local connectedness in paracompact spaces}

7. Let $X$ be a paracompact space.

Definition 7. (cf. [6], p. 153) The space $X$ is said to be semi- $l c_{n}$ if each $x \in X$ has a neighbourhood $U(x)$ such that

$$
\operatorname{Im}\left(H_{n}\left(i^{U(x)}, x^{x}\right)\right)=0 .
$$

We call $X l c_{n}$ if there exists for each $x \in X$, and for each neighbourhood $U(x)$ of $x$, a neighbourhood $V(x)$ in $U(x)$ such that

$$
\operatorname{Im}\left(H_{k}\left(i^{V(x)}, U(x)\right)\right)=0
$$

for each $k<n+1$.

We denote by $\operatorname{Cov}(X)$ the set of open and proper coverings of $X$. The module $H_{n}(\operatorname{Cov}(X))$ is denoted by $\check{H}_{n}(X)(n \in Z)$. If $A$ is a paracompact subspace of $X$, then $\operatorname{Cov}(X, A)$ shall be the set of open and proper coverings of $(X, A)$ and $\check{H}_{n}(X, A)(n \in Z)$ the module $H_{n}(\operatorname{Cov}(X, A))$.

Lemma 2. If $X$ is (semi-)lc $c_{n}$, then $\operatorname{Cov}(X)$ is (semi-) $l c_{n}$.

Proof. If $X$ is semi- $l c_{n}$, we choose for each $x \in X$ an open neighbourhood $U(x)$ satisfying (12). Then every open and proper refinement of $(U(x))_{x \in X}$ is an $n$-refinement of the covering $\{X\}$ of $X$.

If $X$ is $l c_{n}$ and $\alpha \in \operatorname{Cov}(X)$, let $\alpha^{\prime} \in \operatorname{Cov}(X)$ be a star refinement of $\alpha$. Each $x \in X$ has an open neighbourhood $U(x)$, which belongs to $\alpha^{\prime}$, and an open neighbourhood $V(x)$ in $U(x)$ satisfying (13). Then every open and proper refinement of $(V(x))_{x \in X}$ is a strong $n$-refinement of $\alpha$ (cf. [1], p. 320).

Theorem 1 and Lemma 2 imply

Theorem 3. If $X$ is $l c_{n-1}$ and semi- $l c_{n}$, then the canonical homomorphism

$$
t_{p}: H_{p}(X) \rightarrow \check{H}_{p}(X)
$$

is bijective for each $p<n+1$.

If $C(P)$ is a subcomplex of $C(Q)$ for each $P \subset Q \subset X$, then Theorem 2 and Lemma 2 imply 
Theorem 4. If $X$ and $A$ are $l c_{n-1}$ and semi-lch homomorphism

$$
t_{p}: H_{p}(X, A) \rightarrow \check{H}_{p}(X, A)
$$

is bijective for $p<n+1$.

8. Singular homology: Let $C^{A}$ and $C^{\alpha A}(\alpha \in \operatorname{Cov}(X))$ be the singular complexes of the example $1^{\circ}$, pp. 4,5 . In virtue of the homotopy equivalence

$$
H_{n}\left(C^{A}(P)\right) \cong H_{n}\left(C^{\alpha A}(P)\right) \quad(P \subset X, n \in Z),
$$

$C^{A}$ and $C^{\alpha A}$ have the same homology functors $H_{n}(n \in Z)$. Moreover it is easy to see that

$$
\stackrel{\alpha}{C_{p, q}^{\alpha A}}=\stackrel{\alpha}{C_{p, q}^{A}}
$$

for $p>-1$. If $\alpha>\beta \in \operatorname{Cov}(X)$, we obtain the commutative diagram

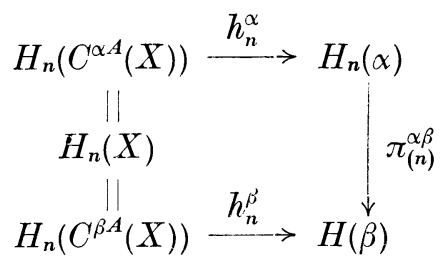

and the limit homomorphism

$$
t_{n}: H_{n}(X) \rightarrow \check{H}_{n}(X)
$$

of the homomorphisms $h_{n}^{\alpha}(\alpha \in \operatorname{Cov}(X))$ for each $n \in Z$. In virtue of the homotopy equivalences (14) and the equations (15) above Lemma $l$ is valid here, and we obtain Theorem 3 for the complex $C^{A}$.

If $P \subset Q \subset X$, then $C^{\theta}(P)$ and $C^{\alpha \theta}(P)$ are subcomplexes of $C^{\theta}(Q)$ and $C^{\alpha \sigma}(Q)$ respectively. In a similar manner Theorem 4 is then obtained for the complex $C^{\sigma}$.

Definition 8. (see [6], p. 153) Let $M$ be the ring $Z$ and $C$ the complex $C^{\emptyset}$. Then $X$ is semi- $q-l c_{s}$ if it is semi- $l c_{q}$, and $l c_{s}^{p}$ if it is $l c_{p}(p, q \in Z)$.

Lemma 3. If $X$ is semi-n-lc $l c_{s}$ and $l c_{s}^{n-1}, A \subset X$ closed and $l c_{s}^{n-1}$, then $X$ is semi- $l c_{n}$ and $l c_{n-1}$ with respect to $C^{A}$ for any module $M$.

Proof: We shall prove first that $X$ is semi- $l c_{n}$ and $l c_{n-1}$ with respect to $C^{\sigma}$ for any module $M$. Let $p$ be an integer, smaller than $n+1$, and $U$ a neighbourhood of $x \in X$. If $p=n$, we assume that $U=X$. Denoting by $H_{q}(P, M, S)$ the module $H_{q}\left(C^{\sigma}(P)\right)(q \in Z, P \subset X)$ we obtain by assumption neighbourhoods $W \subset V \subset U$ of $x$ such that 


$$
\operatorname{Im}\left(H_{p-1}\left(i^{W, V}\right): H_{p-1}(W, Z, S) \rightarrow H_{p-1}(V, Z, S)\right)=0
$$

and

$$
\operatorname{Im}\left(H_{p}\left(i^{V, U}\right): H_{p}(V, Z, S) \rightarrow H_{p}(U, Z, S)\right)=0 .
$$

Setting $H_{p}\left(i^{V, U}\right) \otimes M=a, b=H_{p}\left(i^{W, V}\right): H_{p}(W, M, S) \rightarrow H_{p}(V, M, S)$ $c=H_{p}\left(i^{V \cdot U}\right): H_{p}(V, M, S) \rightarrow H_{p}(U, M, S)$ and $d=\operatorname{Tor}\left(H_{p-1}\left(i^{W, V}\right), M\right): \operatorname{Tor}\left(H_{p-1}(W, Z, S), M\right) \rightarrow \operatorname{Tor}\left(H_{p-1}(V, Z, S), M\right)$, we obtain the commutative diagram $0 \rightarrow H_{p}(W, Z, S) \otimes M \rightarrow H_{p}(W, M, S) \rightarrow \operatorname{Tor}\left(H_{p-1}(W, Z, S), M\right) \rightarrow 0$

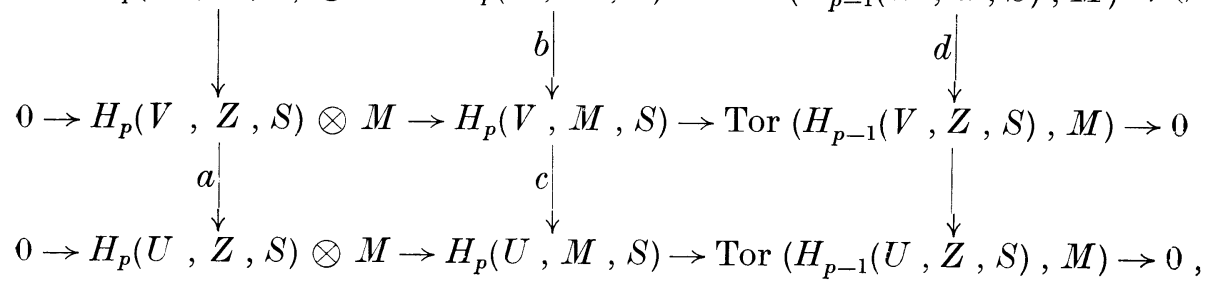
with exact rows and $a=d=0$. Simple diagram chasing yields $c b=0$, which proves the assertion.

With $U$ given as above, by assumption we may choose the neighbourhoods $W \subset V \subset U$ of $x$ such that

and

$$
\operatorname{Im}\left(H_{p}\left(i^{V, U}\right): H_{p}(V, M, S) \rightarrow H_{p}(U, M, S)\right)=0,
$$

$$
\operatorname{Im}\left(H_{p-1}\left(i^{W \cap A, V \cap A}\right): H_{p-1}(W \cap A, M, S) \rightarrow H_{p-1}(V \cap A, M, S)\right)=0 .
$$

Writing $a=H_{p}\left(i^{V, U}\right)$ and $c=H_{p-1}\left(i^{W \cap A . V \cap A}\right)$ above, $b=H_{p}\left(i^{W, V}\right)$ : $H_{p}\left(C^{A}(W)\right)=H_{F}(W, W \cap A, M, S) \rightarrow H_{p}(V, V \cap A, M, S)=H_{p}\left(C^{A}(V)\right)$ and $d=H_{p}\left(i^{V, U}\right): H_{p}\left(C^{A}(V)\right)=H_{p}(V, V \cap A, M, S) \rightarrow H_{p}(U, U \cap A, M, S)$ $=H_{p}\left(C^{A}(U)\right)$, we obtain the commutative diagram

$\ldots \rightarrow H_{p}(W, M, S) \rightarrow H_{p}(W, W \cap A, M, S) \rightarrow H_{p-1}(W \cap A, M, S) \rightarrow \ldots$

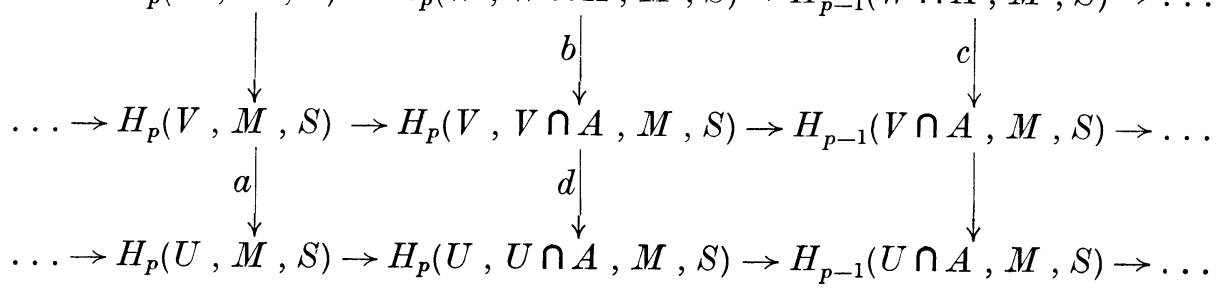

with exact rows and $a=c=0$. Diagram chasing yields $d b=0$, which completes the proof. 
Theorem 4 then contains a new proof of Theorem 1 of Mardešić in [6]. If $A$ is closed in $X$, then Theorem 3 and Lemma 3 yield a slight generalization of it.

9. In a compact space $X$ the results of section 7 can be applied to the net $\operatorname{Cov}^{f}(X)$ of finite open and proper coverings of $X$ and to the complex $C^{\Sigma}$ of example $2^{\circ}$ on page 6 above. If $X$ is $l c_{n-1}$ and semi- $l c_{n}$ with respect to $C^{\Sigma}$, it follows that the canonical homomorphism

$$
t_{p}: H_{p}(\Sigma, M)=H_{n}(X) \rightarrow \check{\check{H}}_{p}(X)=\check{\check{H}}_{p}(\Sigma, M)
$$

is bijective for $p<n+1$. This result may be added to Lemma 10 of [3], p. 18.

\section{Appendix}

Corrections to the author's paper [3]:

\begin{tabular}{|c|c|c|c|c|c|c|c|}
\hline page & line & for & read & page & $\operatorname{line}$ & for & read \\
\hline \multirow[t]{3}{*}{13} & 25 & $H^{j}$ & $H^{n}$ & 33 & fig. 4 & $\bar{\beta}_{1}$ & $\beta_{1}$ \\
\hline & 32 & $\varphi$ & $\varphi_{*}$ & 35 & 8 & $A, B$ & $\bar{A}, B$ \\
\hline & 33 & $\varphi$ & $\varphi^{*}$ & 36 & 16 & $\bar{A} \cup \bar{B}$ & $\bar{A} \cap \bar{B}$ \\
\hline 14 & 29 & $\S 6$ & $\S 1$ & 37 & 16 & $\AA$ & $\overline{8}$ \\
\hline 16 & \multicolumn{3}{|c|}{$\begin{array}{l}\text { Example D. see p. } 7 \\
\text { above }\end{array}$} & & \multicolumn{3}{|c|}{$\begin{array}{c}19 \text { for } C^{p}\left(\alpha, C^{q}, C^{q}\left(\beta, \stackrel{\circ}{N^{i}}\right)\right) \\
\quad \operatorname{read} C^{p}\left(\alpha, C^{q}\left(\beta, \stackrel{\circ}{N}^{i}\right)\right)\end{array}$} \\
\hline \multirow[t]{2}{*}{19} & 1 & $\pi_{(n)}^{\gamma}$ & $\tilde{\pi}_{(n)}^{\gamma}$ & & 19 & $\S 6$ & $\S_{i} 1$ \\
\hline & 3 & $\check{\pi}_{(n)}^{\gamma}$ & $\pi_{(n)}^{\gamma}$ & 39 & 2 & $" Z^{-1 *}$ & $\begin{array}{l}" Z^{--1 *} \\
-1\end{array}$ \\
\hline \multicolumn{4}{|c|}{ for $\varepsilon_{(n)}=\pi_{(n)}^{\gamma}\left(\tilde{\pi}_{(n)}^{\gamma}\right)^{-1}$} & 41 & 12 & $\begin{array}{l}-1 \\
S^{-1}\end{array}$ & $\bar{S}$ \\
\hline \multicolumn{4}{|c|}{$\operatorname{read} \varepsilon_{(n)}=\left(\tilde{\pi}_{(n)}^{\gamma}\right)^{-1} \pi_{(n)}^{\gamma}$} & 43 & 2 & $A^{p}$ & $A_{p}$ \\
\hline 20 & $\begin{array}{r}6 \\
19\end{array}$ & $\begin{array}{l}g^{j-1} \\
A_{j-i}^{j-i}\end{array}$ & $\begin{array}{c}g_{j-1}^{j-1} \\
A_{3 j-i}^{j-i}\end{array}$ & & 29 & $A$ & $A_{0}$ \\
\hline 29 & $1,4,5$ & see & above & & & & \\
\hline
\end{tabular}




\section{References}

[1] Floyd, E. E.: Closed coverings in Cech homology theory. - Trans. Amer. Math. Soc. 84, 1957.

[2] Godement, R.: Théorie des faisceaux. - Actualités Sci. Ind. 1251, Paris 1958.

[3] Jussila, O.: Sur les homomorphismes canoniques de Čch. - Ann. Acad. Sci. Fenn. A. I. 326, 1963.

[4] Kelley, J.-Pitcher, E.: Exact homomorphism sequences in homology theory. Ann. of Math. 48, 1947.

[5] Lefschetz, S.: Algebraic topology. - Amer. Math. Soc., New York 1942.

[6] MARDešÍ́, S.: Comparison of singular and Čch homology in locally connected space. - Michigan Math. J. 6, 1959. 